\title{
Fused Filament Fabrication of ONYX-Based Composites Coated with Aluminum Powders: a Preliminary Analysis on Feasibility and Characterization
}

\author{
Alessia Serena Perna, Antonello Astarita, Domenico Borrelli, Antonio Caraviello, Francesco Delloro, Roberta Della \\ Gatta, Patrizio Lomonaco, Ilaria Papa, Raffaele Sansone and Antonio Viscusi
}

\author{
Alessia Serena Perna. Department of Chemical, Materials and Production Engineering, University of Naples Federico II, Piazzale \\ Tecchio 80, Naples, Italy \\ University of Bergamo, Bergamo, Italy \\ Corresponding author: alessia.perna@unibg.it \\ Antonello Astarita. Department of Chemical, Materials and Production Engineering, University of Naples Federico II, Piazzale \\ Tecchio 80, Naples, Italy \\ Domenico Borrelli. Sophia High tech, Via Romani 228, 80048, Sant'Anastasia NA, Italy \\ Antonio Caraviello. Sophia High tech, Via Romani 228, 80048, Sant'Anastasia NA, Italy \\ Francesco Delloro. Département Mécanique et Matériaux (MINES ParisTech), Paris, France \\ Roberta Della Gatta. Department of Chemical, Materials and Production Engineering, University of Naples Federico II, Piazzale \\ Tecchio 80, Naples, Italy \\ Patrizio Lomonaco. Département Mécanique et Matériaux (MINES ParisTech), Paris, France \\ Ilaria Papa. Department of Chemical, Materials and Production Engineering, University of Naples Federico II, Piazzale Tecchio 80, \\ Naples, Italy \\ Raffaele Sansone. Sophia High tech, Via Romani 228, 80048, Sant'Anastasia NA, Italy \\ Antonio Viscusi. Department of Chemical, Materials and Production Engineering, University of Naples Federico II, Piazzale \\ Tecchio 80, Naples, Italy
}

\begin{abstract}
Polymer-based AM methods are the most mature additive technologies for their versatility and variety of products obtainable. The addition of fibre reinforcement can also confer to the manufactures produced good mechanical properties. Unfortunately, several applications are still precluded because polymers cannot guarantee appropriate electrical conductivity, erosion resistance and operating temperature. Aiming to overcome these issues, the metallization of the surfaces emerges as a possible solution. Unfortunately, thermoplastic polymers exhibit thermosensitive behaviour and run the risk of being damaged when traditional metallization techniques, which require the melting of metal powders which will act as a protective coating. For this reason, studies have focused on Cold Gas Dynamic Spray, an additive manufacturing technology, which exploits kinetic energy to favour the adhesion of metal particles rather than the increase in temperature. In this work, a first attempt is made to verify the feasibility of cold spray coatings on 3D printed composite substrates, produced by means of Fused Filament Fabrication (FFF) technique. FFF technology allows the deposition of two different types of filaments by using a double extruder. These composite fibres within 3D printed parts manage to give the object a resistance comparable to that of a metal part with lower production cost and a high degree of automation. These structures, made of ONYX, a Nylon matrix in which short carbon fibres are dispersed, and reinforced with long carbon fibres, are designed to better fit the CS deposition. Aluminium coatings have been produced and a characterization campaign has been carried on.
\end{abstract}

Keywords. Cold Spray, Composite, Aluminium Powders, Additive Manufacturing

\section{Introduction}

In the last several years, additive manufacturing technologies are polarizing the interest of industrial and scientific world due to unique characteristics and the possibility to suitably tailor the products manufactured [1]. The possibility to produce complex components in a single step and with limited human intervention required during the process offers a plethora of possibility not yet exploited with other technologies. One of the most widespread classes of 
materials employed in 3D printing technologies are the thermoplastic polymers, due to their workability and reduced melting temperature, an indispensable feature required for the majority of printing equipments, that exploit the thermal energy obtained by means of electrical resistance [2,3]. In fact, one of the most widespread techniques for polymer-printing is the Fused Filament Fabrication, where thermoplastic filaments are extruded by means of a remotecontrolled heated nozzle. Recently more interest has been focused on applying FFF technologies for printing composite material by making use of a double nozzle, in order to alternatively printing the matrix material and the reinforcement fibres to obtain lightweight and high resistance components with reduced production time and higher precision and automation compared to traditional technologies [4]. However, the products manufactured still suffers from the major drawback that has limited the diffusion of composite materials such as low thermal and electrical conductivity and poor wear and scratch behaviour [5]. For this reason, hybrid composite/metallic structures have been developed in order to improve the surface characteristics of the component, although the most common metallization techniques have several disadvantages and may lead to severe surface degradation due to the high temperatures applied [6,7]. In order to overtake those issue, a relatively new technology, previously applied mostly on metallic surfaces, emerges: the cold spay technology. Gas dynamic cold spraying (CGDS) or cold spraying (CS) method is the newest among all known thermal spraying technologies. This technology allows the deposition of micrometric particles (with diameters in the order of magnitude 10-100 $\mu \mathrm{m}$ ) with temperatures well below their melting point [8]. In fact, this technology exploits the kinetic energy obtained by accelerating the particles by means of a carrier gas rather than using a thermal source to produce the coating. The flow of micrometric powders $(1-50 \mu \mathrm{m})$ is accelerated by the supersonic flow of a compressed gas.

The deposition process is "Cold" due to the short contact time between particles and hot gas, of the order of nanoseconds, and the rapid cooling of the gas during expansion in the divergent section of the nozzle. Inert gases or air and convergent-divergent 'DeLaval' nozzles are used to accelerate solid metallic particles at supersonic speeds (300 - $1200 \mathrm{~m} / \mathrm{s})$, so the result of the impact of particles against the substrate makes adhesion possible and coating formation. In fact, at the moment of impact with the substrate, the particles undergo a high plastic deformation which causes a mechanical anchorage and therefore the growth of the deposit on the substrate. This results in lower degradation of the processed surface while the sprayed particles retain their production characteristics. Hence, this technology is particularly suitable for temperature-sensitive materials, like thermoplastic-based composite, serving possibly as a worthy ally when applied on 3D-printed materials $[9,10]$. For this reason, this work deals with the opportunity of coupling FFF technology and CS technology in order to new high-performance, tailored products.

After assessing the feasibility of CS coatings on additive manufactured composites in a previous work of this research group, in this paper a further step forward has been made: three different types of FFF-printed laminates have been produced, by changing the thickness of matrix on the surface of the composites in order to assess the influence of the lay-up sequence on the deposition and overall quality of the laminates. Each laminate was then coated with micrometric aluminium powders by means of low-pressure cold spray equipment by changing the number of passes and analysing the growth and densification of the coating. The obtained laminates were then characterised through electronics and confocal analyses.

\section{Materials and Methods}

\subsection{D-printed laminates production}

Onyx-matrix reinforced with carbon fibres were produced for this work. Onyx is a novel nylon-based material produced by Markforged reinforced with short carbon fibres. This material is characterized by high strength, high resistance and excellent workability, making it a perfect choice for the 3D printing, also considering its heath deflection temperature of $140^{\circ} \mathrm{C}$ and the low thermal deformation guaranteed by its short-fibre reinforcement [11]. The laminates are reinforced 
with long carbon fibre, optimized for the printing process: a thin layer of thermoplastic material surround each packet of fibres serving as an interlayer between fibres and matrix. The fibers were deposited layer by layer guaranteeing high performances and design freedom. The laminates were produced with the Fused Filament Fabrication technology, by means of a MarkForged X7 3D printer. The presence of a double extruder on the machine (allowing a Continuous Fibre Fabrication process), allow to generate the laminate one layer at time by alternating fibre and matrix layers: the first nozzle deposit the thermoplastic matrix while the second extruder wrap the fibre, guaranteeing an high degree of precision ( $1 \mu \mathrm{m}$ ) thanks to a built-in laser micrometre capable of scanning the process plate and build a map of its surface. This allow to continuously calibrate the height of the nozzle during the printing process. Three typologies of laminates were produced, characterized by a different number of matrix layers on the surface of the specimen. Each matrix superficial layer has a $0.125 \mathrm{~mm}$ thickness. The surface area of the panels is $5 \times 5 \mathrm{~cm}$ and an overall thickness varies from $2 \pm 0.2 \mathrm{~mm}$, for the single matrix-layer panel, to $2.5 \pm 0.2 \mathrm{~mm}$ for the thickest one. The lay-up sequence applied for all the panels is illustrated in Fig.1.

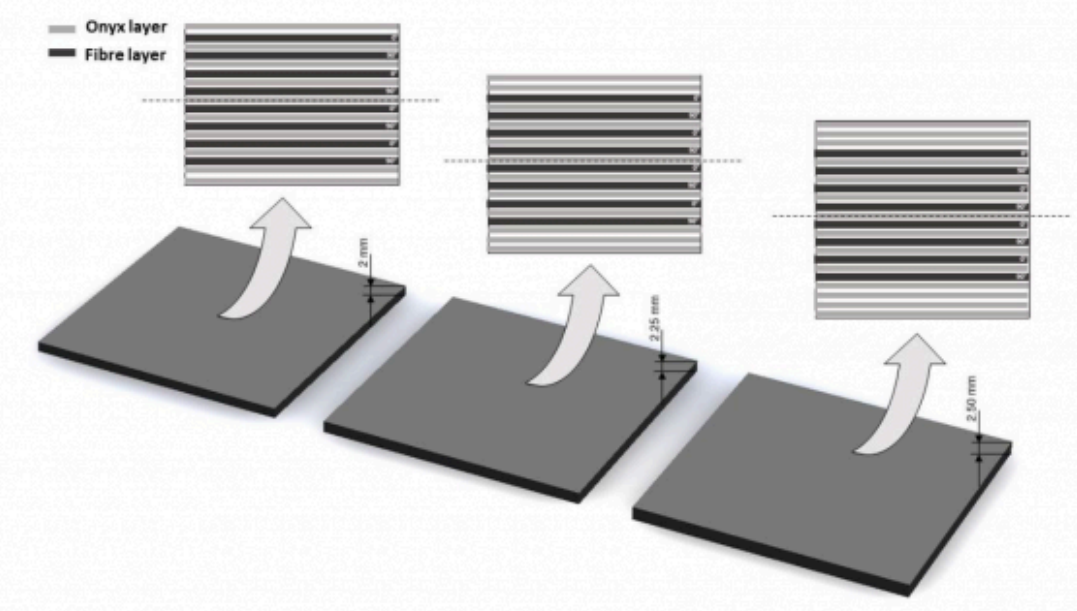

Fig. 1. Layup sequence of the printed laminates

It should be noted that fibre layers are alternated with matrix layers. The infill strategy chosen for the matrix is a triangular one for the matrix in the core, while it follows a 45 degrees pattern for the matrix on the surface, as shown in Fig.2 


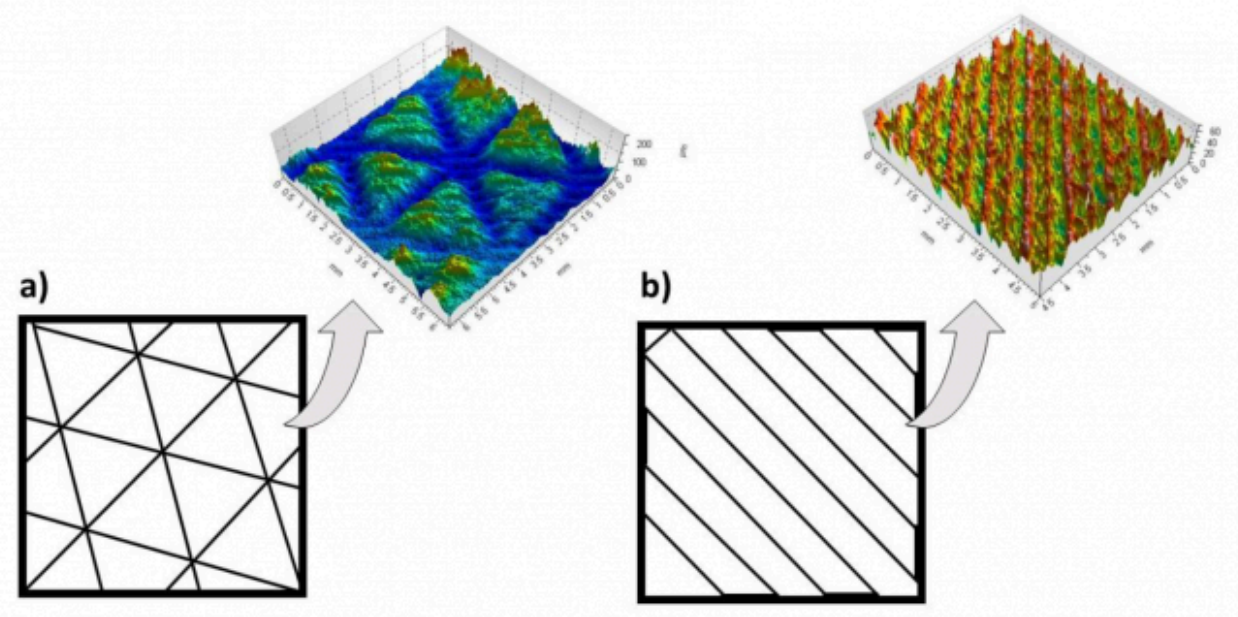

Fig. 2. Infill strategy of the matrix: a) matrix in the core of the laminate b) matrix on the surface

\subsection{Cold Spray process}

Micron-sized aluminium particles were deposited on the printed laminates employing a low-pressure CS equipment (Dycomet 423), using nitrogen as a carrier gas. Low-pressure process exploit a manoeuvrable and light "gun", conversely to the larger apparatus used in the high-pressure process and, for this reason, they are typically portable and are limited to 300-600 m/sec particle velocities. They are used in the application of lighter materials and they generally utilize readily available air or nitrogen as propellant gases. The particles (provided by Toyo aluminium) have a mean diameter of $20 \mu \mathrm{m}$ and a spherical shape. The process parameters and powder characteristics were chosen due to the previous studies developed on the subject [12-14] and are summarized in Table 1.

Table 1. CS process parameters and materials

\begin{tabular}{c|c|c|c|c}
\hline $\begin{array}{c}\text { Stand-off } \\
\text { distance }\end{array}$ & Gas typology & $\begin{array}{c}\text { Inlet gas } \\
\text { pressure }\end{array}$ & $\begin{array}{c}\text { Inlet gas } \\
\text { temperature }\end{array}$ & $\begin{array}{c}\text { Spraying } \\
\text { gun speed }\end{array}$ \\
\hline $25 \mathrm{~mm}$ & Nitrogen & $6 \mathrm{bar}$ & $250^{\circ} \mathrm{C}$ & $8 \mathrm{~mm} / \mathrm{s}$ \\
\hline
\end{tabular}

On each panel, two rectangular-shaped coatings have been produced: one with a single pass of deposition, the other with a double pass, in order to analyze the growth of the coating and the changes in terms of powders distribution and deformation.

\subsection{Surface characterization}

The surface of the laminates before the deposition was analysed through a confocal microscope (LEICA DCM3D Scan) in order to assess the differences in terms of surface finishing. In fact, a three-dimensional surface of the analysed area was obtained, and profile were extracted in order to calculate the coating height. After the deposition, the surface 
coverage was calculated through ImageJ analyses of the electronic micrographs (obtained through SEM electronic microscope) of the samples.

\section{Results and Discussion}

It was possible, with the parameters chosen, to assess the feasibility of the coating deposition on all the specimen analyzed. In Fig.3, the coated laminates are shown. As evident in the picture, the presence of the coating enhances the visibility of the triangular sub-superficial matrix pattern, especially for the laminates characterized by a lower superficial matrix presence.

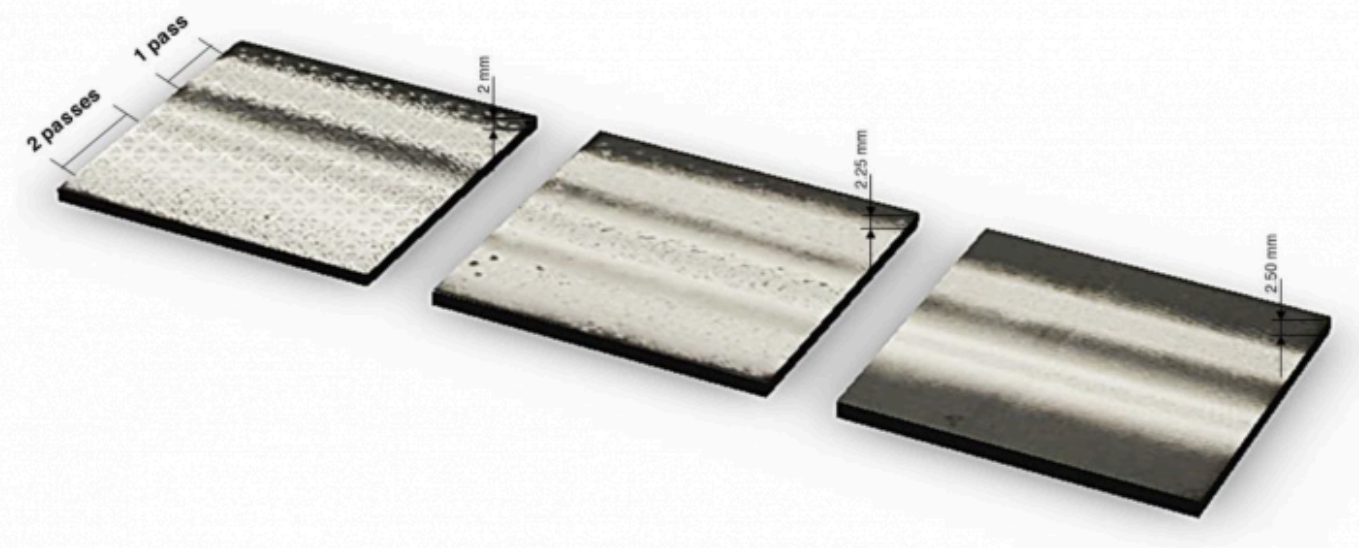

Fig. 3. Coated laminates. Deposition tracks are evident on all the panels. Superficial texturing is enhanced for the $2 \mathrm{~mm}$ panel.

As it is highlighted from a visual inspection, the coating produced appears thick and homogeneous and no apparent degradation of the substrates is noticed. Ulterior analyses have been carried on to assess the best layup sequence for the deposition and the quality of the overall coating. The influence of the sub-superficial pattern on the surface finishing of the laminates was also investigated employing confocal analyses carried out on the panels. The mean coating height was calculated by extracting profiles from the three-dimensional surface obtained. As evidenced in Fig.4, the core matrix pattern has a clear influence on the deposition of the first layer of matrix surface, while is not evident anymore when an ulterior layer of matrix is deposited. The different surface finishing of the laminates cause a different deposition behaviour on the various specimens as evidenced from the profiles analysed. In fact the coating height is evidently higher on the $2.25 \mathrm{~mm}$ specimen, confirming the visual inspections. 
a)

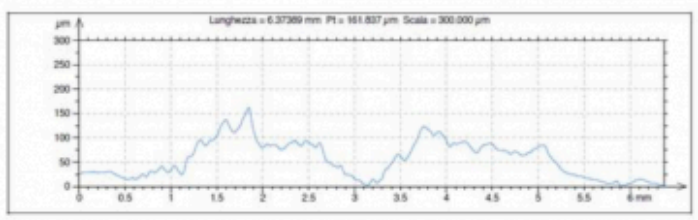

b)

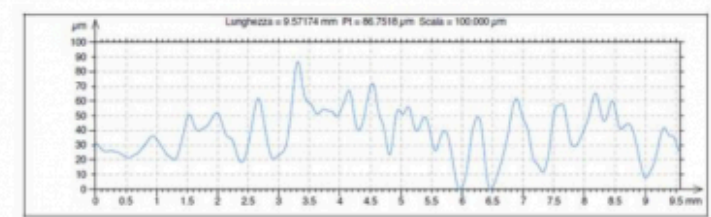

c)
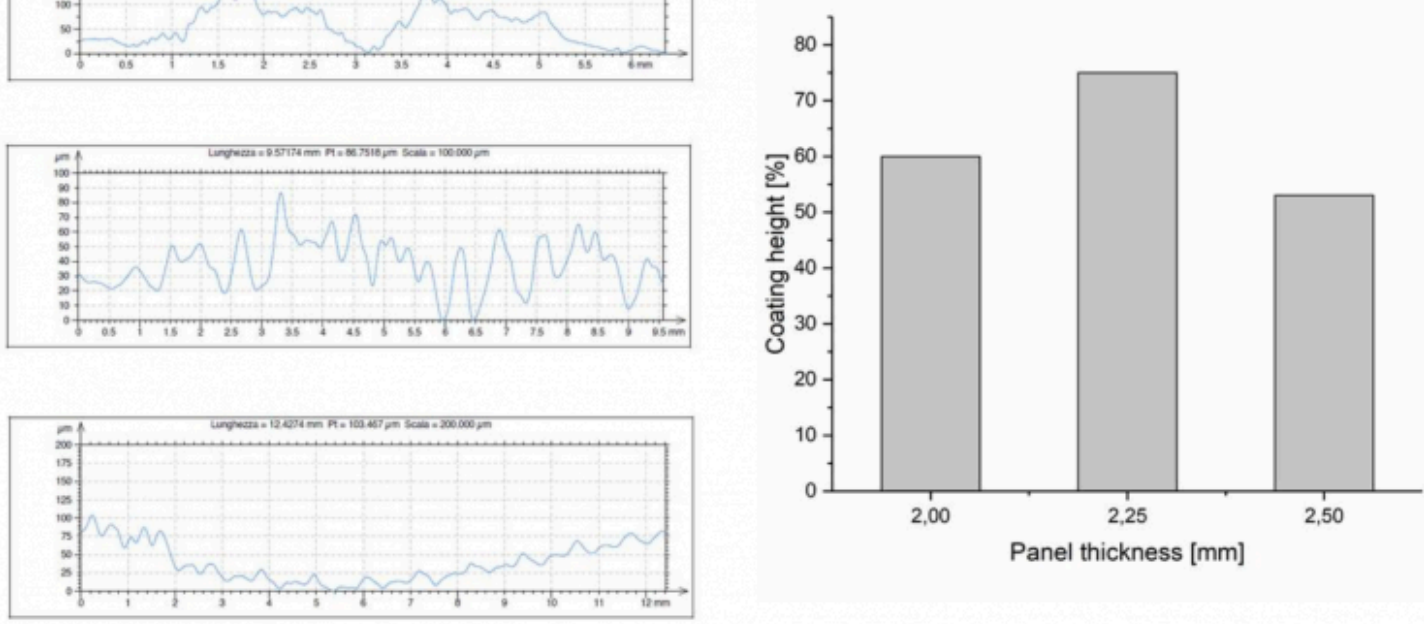

Fig. 4 Extracted specimen profiles and coating height. a) $2 \mathrm{~mm}$ panel, b) $2.25 \mathrm{~mm}$ panel c) $2.50 \mathrm{~mm}$ panel

As appears from the SEM analyses (portrayed in Fig.5) the sprayed particles experience severe deformation upon impact, due to the rigidity of the reinforced substrates. However, observing the single-pass tracks, the deposition is not homogeneous on the panels and several blank spots in the coatings are present. In particular, for the $2 \mathrm{~mm}$ substrate, characterized by fewer matrix layers on the surface the particles does not adhere perfectly to the substrate. This may be caused by the enhanced surface pattern, evidenced in the confocal analyses, that hinder the deposition causing particles to rebound. The best-case appears the intermediate case $(2.25 \mathrm{~mm}$ panels) where the particles are uniformly deposited through all the surface area. For the thickest panel $(2.50 \mathrm{~mm})$ the deposition appears less efficient: this phenomenon may be related to the lower deformation experienced by the particles as the influence of the fibres is less effective due to the thicker matrix surface layer. This may cause a rising of the deposition window, compared to the cases where the fibres have a higher influence, as evidenced in several works present in literature $[14,15]$. 


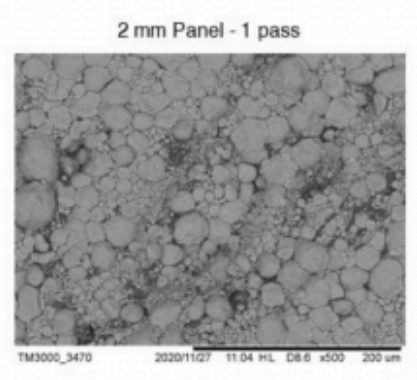

$2 \mathrm{~mm}$ Panel - 2 passes

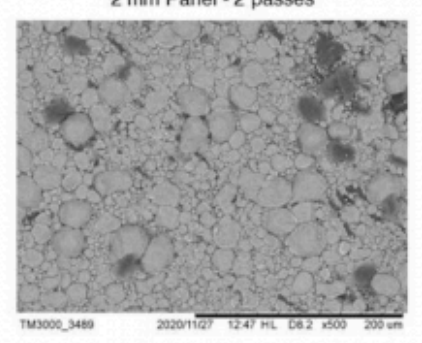

$2.25 \mathrm{~mm}$ Panel - 1 pass

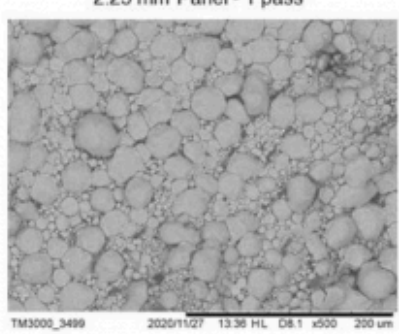

$2.25 \mathrm{~mm}$ Panel - 2 passes

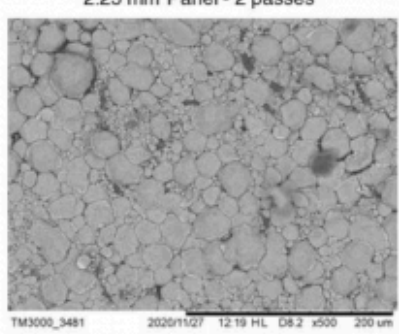

$250 \mathrm{~mm}$ Panel - 1 pass

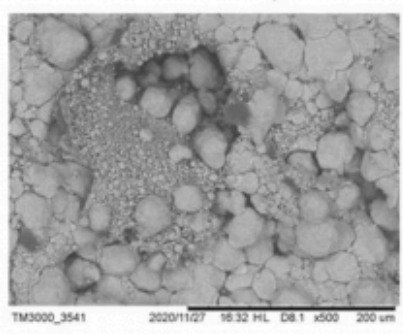

$2.50 \mathrm{~mm}$ Panel - 2 passes

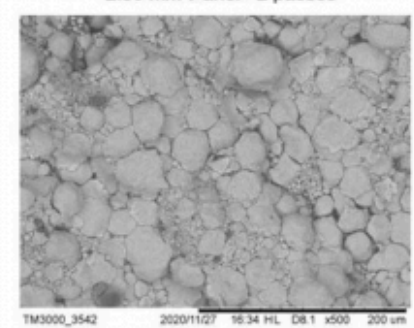

Fig. 5. SEM top view acquisitions of the coating on the different panels.

The surface coverage analyses further confirms those asserts. Those analyses were obtained by analysing the SEM images through ImageJ software obtaining a coating map where the coating particles are evidenced in white, while the voids (e.g. the polymer matrix beneath the surface) are evidenced in black, as shown in Fig.6.

a)

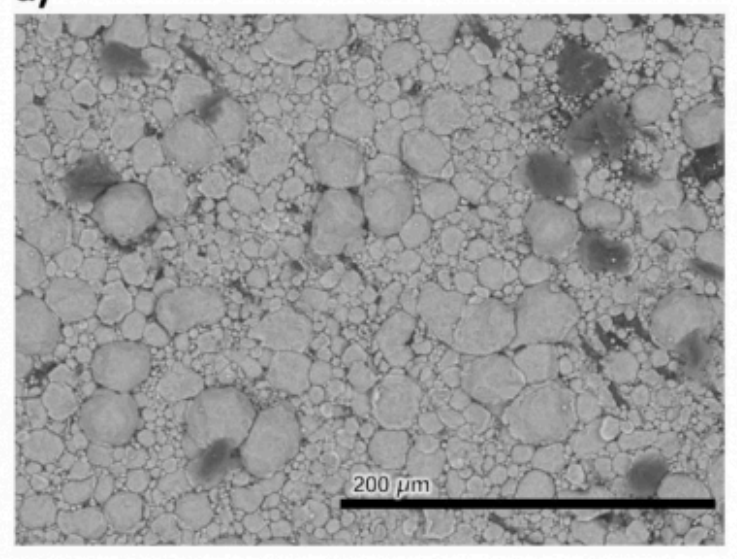

b)

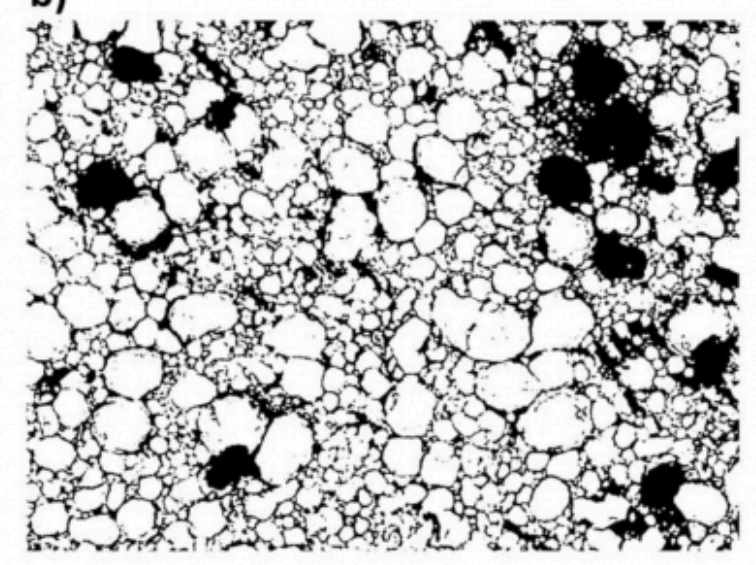

Fig. 6. Coating top view SEM acquisition a) original image b) post-processed image for the calculation of the surface coverage

As regards the double passes tracks, it is evident, for all the panel analyzed, an overall increase of the coverage compared with the respective single-pass coatings obtained on the same panel: this is also confirmed by the surface coverage analyses presented in Fig.7. 


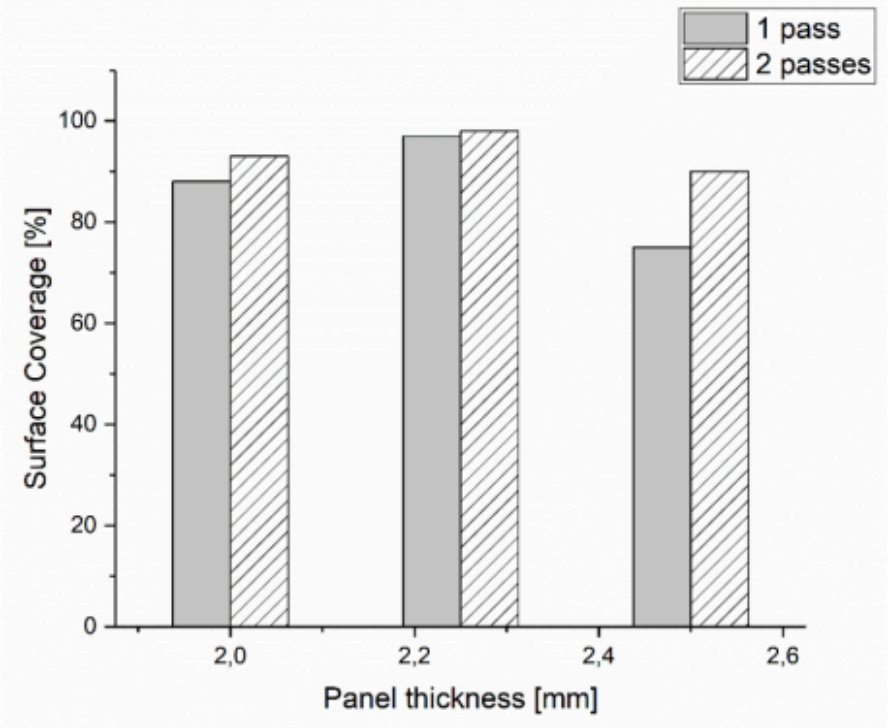

Fig.7. Surface coverage measurements

In fact, a subsequent deposition pass may help to fill the voids left by the first deposition pass. The influence of the surface patterns appears less evident as the number of passes increase.

\section{Conclusions}

The results presented and discussed in the previous segments of this work allow to highlight the following conclusions:

- 3D printed Onyx based laminates reinforced with carbon fibres were successfully produced by means of FFF technology. Three different batches of panels were produces characterized by a different number of matrix layer on the surface. Confocal analyses carried out on the panels confirmed the different surface finishing of the panels produced, differently influenced by the sub-superficial matrix layers patterns.

- Al powders were successfully deposited on the laminates under the established processing conditions; visual inspections and surface coverage were carried out in order to analyse the quality of single pass and double pass coatings.

- The surface finishing of the panels, influenced under a certain limit of substrate matrix layer thickness by the sub-superficial matrix pattern, can influence the deposition. It was also confirmed that fibres can influence particle deformation due to the stiffening of the laminate.

- Further deposition passes may increase the surface coverage of the panels and may mitigate the influence of the sub-superficial matrix layer patterns.

\section{Bibliography}

[1] Das, A., C.A. Chatham, J.J. Fallon, C.E. Zawaski, E.L. Gilmer, C.B. Williams, M.J. Bortner, Current understanding and challenges in high temperature additive manufacturing of engineering thermoplastic polymers. Additive Manufacturing, 2020, 34, 101218. https://doi.org/10.1016/j.addma.2020.101218. 
[2] Blok, L.G., M.L. Longana, H. Yu, B.K.S. Woods, An investigation into 3D printing of fibre reinforced thermoplastic composites. Additive Manufacturing, 2018, 22, 176-186. https://doi.org/10.1016/j.addma.2018.04.039.

[3] González-Henríquez, C.M., M.A. Sarabia-Vallejos, J. Rodriguez-Hernandez, Polymers for additive manufacturing and 4D-printing: Materials, methodologies, and biomedical applications. Progress in Polymer Science, 2019, 94, 57-116. https://doi.org/10.1016/j.progpolymsci.2019.03.001.

[4] Brenken, B., E. Barocio, A. Favaloro, V. Kunc, R.B. Pipes, Fused filament fabrication of fiber-reinforced polymers: A review. Additive Manufacturing, 2018, 21, 1-16. https://doi.org/10.1016/j.addma.2018.01.002.

[5] Wang, X., M. Jiang, Z. Zhou, J. Gou, D. Hui, 3D printing of polymer matrix composites: A review and prospective. Composites Part B: Engineering, 2017, 110, 442-458. https://doi.org/10.1016/j.compositesb.2016.11.034.

[6] White, J., C. Tenore, A. Pavich, R. Scherzer, S. Stagon, Environmentally benign metallization of material extrusion technology 3D printed acrylonitrile butadiene styrene parts using physical vapor deposition. Additive Manufacturing, 2018,. https://doi.org/10.1016/j.addma.2018.05.016.

[7] Su, X., X. Li, C.Y.A. Ong, T.S. Herng, Y. Wang, E. Peng, J. Ding, Metallization of 3D Printed Polymers and Their Application as a Fully Functional Water-Splitting System. Advanced Science, 2019,. https://doi.org/10.1002/advs.201801670.

[8] Hanft, D., J. Exner, M. Schubert, T. Stöcker, P. Fuierer, R. Moos, An overview of the Aerosol Deposition method: Process fundamentals and new trends in materials applications. Journal of Ceramic Science and Technology, 2015,. https://doi.org/10.4416/JCST2015-00018.

[9] Ganesan, A., M. Yamada, M. Fukumoto, Cold spray coating deposition mechanism on the thermoplastic and thermosetting polymer substrates. Journal of Thermal Spray Technology, 2013, 22, 1275-1282. https://doi.org/ 10.1007/s11666-013-9984-x.

[10] Champagne, V., D. Helfritch, The unique abilities of cold spray deposition. International Materials Reviews, 2016, 61, 437-455. https://doi.org/10.1080/09506608.2016.1194948.

[11] Kabir, S.M.F., K. Mathur, A.F.M. Seyam, A critical review on 3D printed continuous fiber-reinforced composites: History, mechanism, materials and properties. Composite Structures, 2020,. https://doi.org/10.1016/ j.compstruct.2019.111476.

[12] Viscusi, A., M. Durante, A. Astarita, L. Boccarusso, L. Carrino, A.S. Perna, Experimental evaluation of metallic coating on polymer by cold spray, in: Procedia Manufacturing, Elsevier B.V., 2020,: pp. 761-765. https://doi.org/10.1016/ j.promfg.2020.04.232.

[13] Perna, A.S., A. Viscusi, A. Astarita, L. Boccarusso, L. Carrino, M. Durante, R. Sansone, Manufacturing of a Metal Matrix Composite Coating on a Polymer Matrix Composite Through Cold Gas Dynamic Spray Technique. Journal of Materials Engineering and Performance, 2019, 28, 3211-3219. https://doi.org/10.1007/s11665-019-03914-6.

[14] Gatta, R. Della, A. Viscusi, A.S. Perna, A. Caraviello, A. Astarita, R. Della Gatta, S. Perna, Cold spray process for the production of AlSi10Mg coatings on glass fibers reinforced polymers. 2020,. https://doi.org/10.1080/ 10426914.2020 .1813895 .

[15] Viscusi, A., A.S. Perna, A. Astarita, L. Boccarusso, A. Caraviello, L. Carrino, R. Della Gatta, M. Durante, R. Sansone, Experimental Study of Cold Sprayed Metallic Coatings on Thermoplastic Matrix Composites. Key Engineering Materials, 2019, 813, 68-73. https://doi.org/10.4028/www.scientific.net/kem.813.68. 
Fused Filament Fabrication of ONYX-Based Composites Coated with Aluminum Powders: a Pre...

PDF automatically generated on 2021-05-20 08:23:25

Article url: https://popups.uliege.be/esaform21/index.php?id=4017

published by ULiège Library in Open Access under the terms and conditions of the CC-BY License

(https://creativecommons.org/licenses/by/4.0) 